\title{
Le ribosome bactérien : structure et fonctions
}

Le ribosome est une particule ribonucléoprotéique, complexe jouant le rôle d'«unité centrale » de la synthèse protéique et relativement conservé au cours de l'évolution. Chez $E$. Coli, modèle le mieux connu, la particule ribosomale de $70 S$ est composée de deux sous-unités de $30 \mathrm{~S}$ et de $50 \mathrm{~S}$. La petite sousunité contient une molécule d'ARN et 21 polypeptides, la grande sous-unité contient deux molécules d'ARN et 32 polypeptides. La combinaison de méthodes immunologiques, morphologiques, physiques, biochimiques et informatiques d'analyse a permis de proposer un modèle assez précis de la structure du ribosome, une fonction particulière pouvant être attribuée aux différentes régions et aux différents constituants de cette particule. C'est la grande sous-unité en tant que telle (et peut-être particulièrement sa molécule d'ARN 23S) qui semble le support de l'activité enzymatique de peptidyl-transférase par laquelle s'établissent les liaisons peptidiques entre les acides aminés.

\section{Mario Di Giambattista Carlo Cocito}

\section{ADRESSE}

M. Di Giambattista: chercheur du Fond National de la Recherche Scientifique. C. Cocito: professeur ordinaire à l'université de Louvain. Unité de microbiologie générale et de génétique moléculaire, faculté de médecine, ICP-UCL 7449, avenue Hippocrate 75,

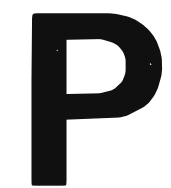

armi les nombreuses caractéristiques de tout être vivant, il en est une particulièrement évidente : la spécificité des structures qu'il est capable non seulement d'édifier mais aussi de transmettre. Le matériel génétique (ADN), qui assure la transmission des caractères d'un individu à l'autre, est responsable des variations héréditaires qui commandent l'évolution, dirige la régulation des nombreuses fonctions cellulaires et contrôle l'édification des protéines. Ces dernières sont constituées d'acides aminés reliés entre eux par des liens peptidiques. La formation du lien peptidique est, chimiquement parlant, une réaction relativement simple. Toutefois, les protéines sont construites à partir de vingt acides aminés différents, suivant un ordre bien défini, dicté par le patrimoine génétique. Cela justifie la complexité du processus de traduction, qui se déroule au niveau d'un organite cellulaire tout aussi complexe : le ribosome. Comme c'est souvent le cas en biologie moléculaire, la bactérie $E$. coli est aussi l'organisme de référence dans l'étude du ribosome. La synthèse protéique se déroule en trois étapes : le démarrage, l'élongation et la terminaison (figure 1). Le démarrage débute par la fixation de l'ARNm à la sous-unité $30 \mathrm{~S}$ du ribosome, suivie par celle de l'aminoacylARNt initiateur, porteur de la formylméthionine (l'anticodon du fMet-ARNt s'appariant avec le codon AUG de l'ARNm). Ces réactions sont 
catalysées par les facteurs de démarrage (IFl, IF2, IF3) qui sont recyclés lors de la liaison de la grande sousunité ribosomale (figure 1). Cette étape de démarrage permet le positionnement du fMet-ARNt au site donneur (site $\mathrm{P}$ ou peptidyl) de la sous-unité $50 \mathrm{~S}$.

L'élongation des chaînes polypeptidiques consiste en trois réactions : (1) la liaison d'un aminoacyl-ARNt (AA-ARNt) ; (2) la formation du lien peptidique; et (3) la translocation. Un second ARNt, porteur de l'acide aminé correspondant au second codon de l'ARNm, se fixe au site receveur (site $\mathrm{A}$ ou aminoacyl) de la sous-unité $50 \mathrm{~S}$. La formation du lien peptidique met en jeu le groupement $\alpha-\mathrm{NH}_{2}$ de l'acide aminé placé au site $\mathrm{A}$ et le groupement - $\mathrm{COOH}$ du peptidyl (ou de la fMet) placé au site P. La translocation du peptidylARNt, du site A vers le site $P$, se traduit par le mouvement du ribosome le long de l'ARNm jusqu'au codon suivant. Les réactions (1) et (3) sont catalysées, respectivement par les facteurs d'élongation EF-Tu et EF-G, l'hydrolyse du GTP fournissant l'énergie nécessaire. La formation de la liaison peptidique est, quant à elle, favorisée par la peptidyl-transférase, activité enzymatique de la grande sous-unité ribosomale. Ce cycle de réactions se répète autant de fois qu'il y a d'acides aminés dans la protéine à assembler.

La terminaison a lieu lorsque le ribosome rencontre un des trois codons de ponctuation (UAG, UAA ou UGA). Grâce à l'intervention des facteurs de terminaison $\left(\mathrm{RF}_{1}, \mathrm{RF}_{2}\right.$, $\mathrm{RF}_{3}$ ), le complexe d'élongation se dissocie; la libération du polypeptide, toujours lié au dernier ARNt, requiert paradoxalement l'intervention de la peptidyl-transférase.

Le but de cet article est de résumer les progrès réalisés, ces dernières années, au niveau de la connaissance de l'architecture des différents constituants du ribosome, ainsi que la manière dont les divers centres actifs s'intègrent au sein de la structure globale de la particule.

\section{Les constituants ribosomaux}

Le ribosome est un complexe ribonucléoprotéique formé de deux sous$\mathrm{m} / \mathrm{s} n^{\circ} 9$ vol. 5 , novembre 89 unités de forme et de taille différentes, désignées par leur coefficient de sédimentation. Les bactéries, les cyanophycées et certains organites cellulaires comme les chloroplastes et les mitochondries renferment des ribosomes du type $70 \mathrm{~S}$ (sous-unités $30 \mathrm{~S}$ et $50 \mathrm{~S})$. En revanche, les cellules eucaryotiques (plantes ou animaux) possèdent des ribosomes dont le coefficient de sédimentation est de $80 \mathrm{~S}$ (sous-unités $40 \mathrm{~S}$ et $60 \mathrm{~S}$ ). La complexité de leur structure réside dans le grand nombre de constituants qui les composent. En effet, plus de 53 protéines différentes ont été identifiées dans le ribosome de $E$. coli [1, 2]. Les protéines ribosomales sont désignées d'une lettre, suivie d'un

\section{${ }^{*}$ GLOSSAIRE*}

ARNm : ARN messager. ARNr: $A R N$ ribosomal. $\boldsymbol{A R N}:$ : ARN de transfert. AA-ARNt: aminoacyl-ARN de transfert.

$E \boldsymbol{F}$ : facteur d'élongation.

GTP : guanosine 5'-triphosphate. EF-G : facteur d'élongation $G$. EF-Tu: facteur d'élongation Tu. fMet : formylméthionine.

fMet-ARNt: l'ARN de transfert porteur de la formylméthionine. $\boldsymbol{R F}$ : facteur de terminaison.

Boucle V: portion de la structure secondaire de l'ARNr $23 S$.

IF : facteur de démarrage.

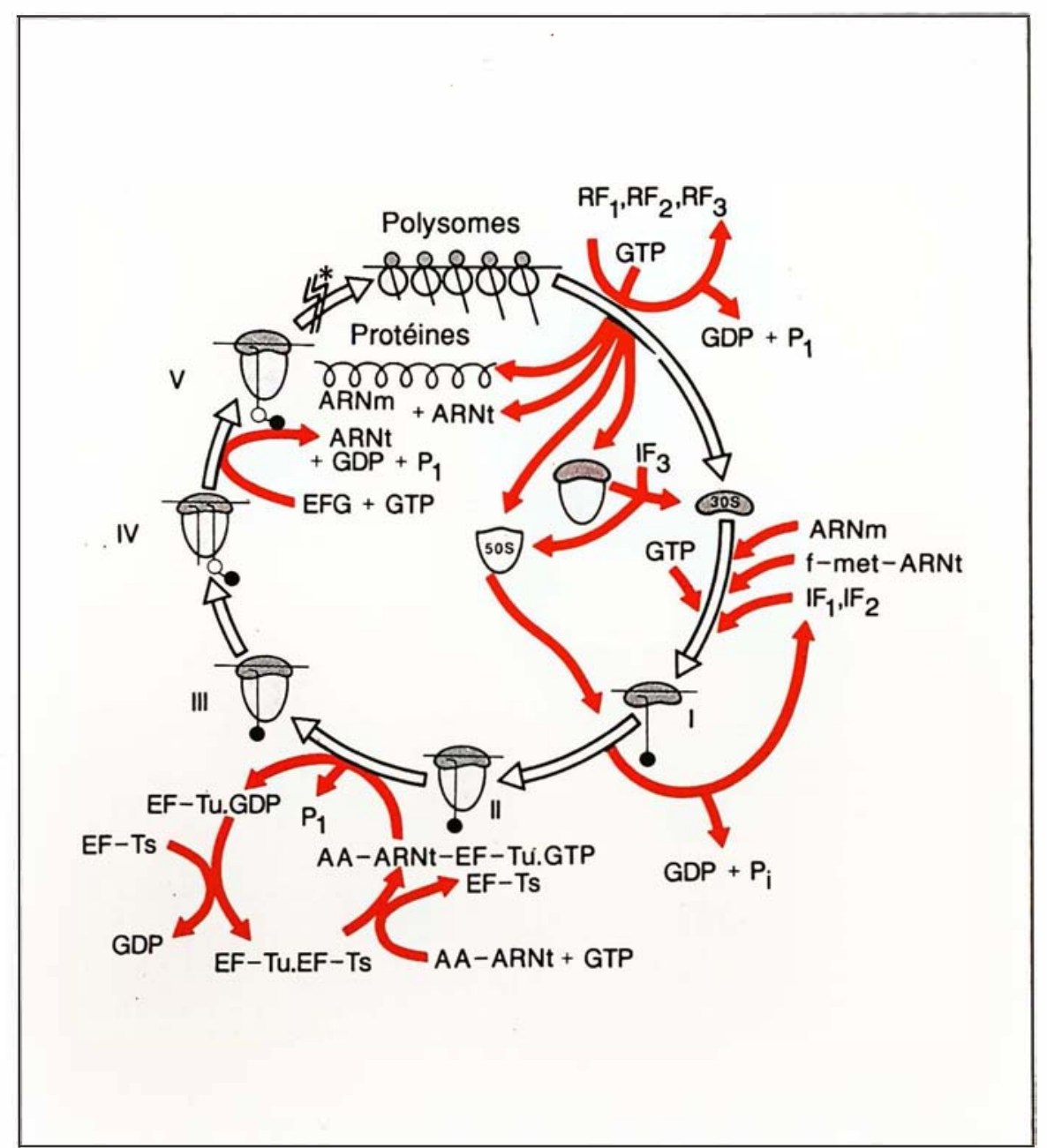

Figure 1. Le cycle ribosomal. I et II: formation du complexe de démarrage ; III : liaison des ARNt (première étape de l'élongation); IV : formation du lien peptidique (deuxième étape de l'élongation); $V$ : translocation (troisième étape de l'élongation); * répétition des étapes III à V. 


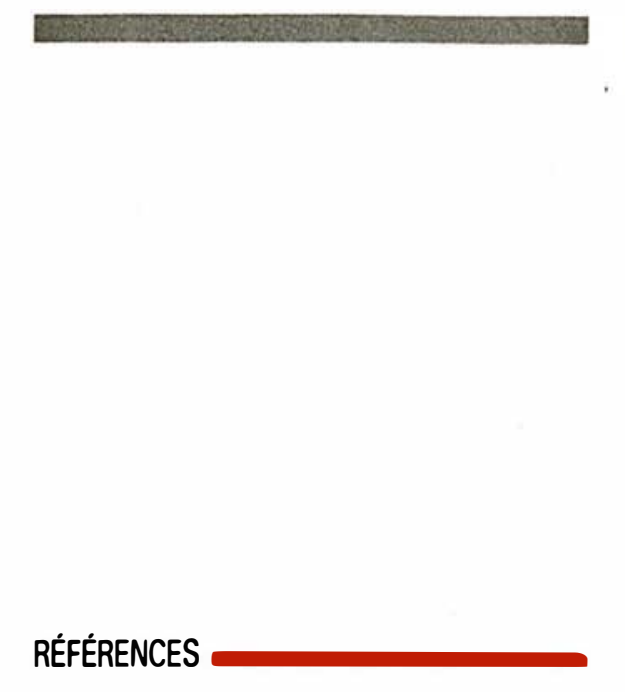

1. Wittmann HG. Components of bacterial ribosomes. Ann Rev Biochem 1982 ; 51 : 15583.

2. Giri L, Hill WE, Wittmann HG. Ribosomal proteins: their structure and spatial arrangement in prokariotic ribosomes. $A d v$ Prot Chem 1984 ; 36 : 1-78.

3. Hardesty B, Kramer G. Structure, function and genetics of ribosomes. New York : Springer-Verlag, 1986

4. Sequences supplement. Nucleic Acids Res 1988 ; 16 (suppl).

5. Woese CR. Bacterial evolution. Microbiol Rev 1987 ; $221-71$.

6. Noller HF. Structure of ribosomal RNA. Ann Rev Biochem 1984 ; 53 : 119-62.

7. Maly P, Brimacombe R. Refined secondary structure models for the 16 and $23 S$ ribosomal RNA of E. coli. Nucleic Acids Res 1983; 11 7263-86.

8. Ebel JP, Branlant C, Carbon P, et al. Structure of ribosomal RNA. In : Helene C, ed. Structure, dynamics, interactions and evolution of biological micromolecules. Boston Reidel D, 1983 : 177-83.

9. Guttel RR, Weiser B, Woese CR, Nol ler HF. Comparative anatomy of $16 \mathrm{~S}$-like ribosomal RNA. Prog Nucleic Acid Res Mol Biol 1985 ; 32 : 155-216.

10. Brimacombe R, Maly P, Zwieb C. The structure of ribosomal RNA and its organisation relative to ribosomal protein. Prog Nucleic Acid Res Mol Biol 1983 ; 28 : 1-48.

11. Kastner B, Stoffler-Meilicke M, Stoffler G. Arrangement of the subunits in the ribosome of $E$. coli: demonstration in immuno electron microscopy. Proc Natl Acad Sci USA 1981 ; 78 : 6652-6.

12. Lake JA. Evolving ribosome structure domains in archaebacteria, eubacteria, eocytes and eukaryotes. Ann Rev Biochem 1985; 54 :

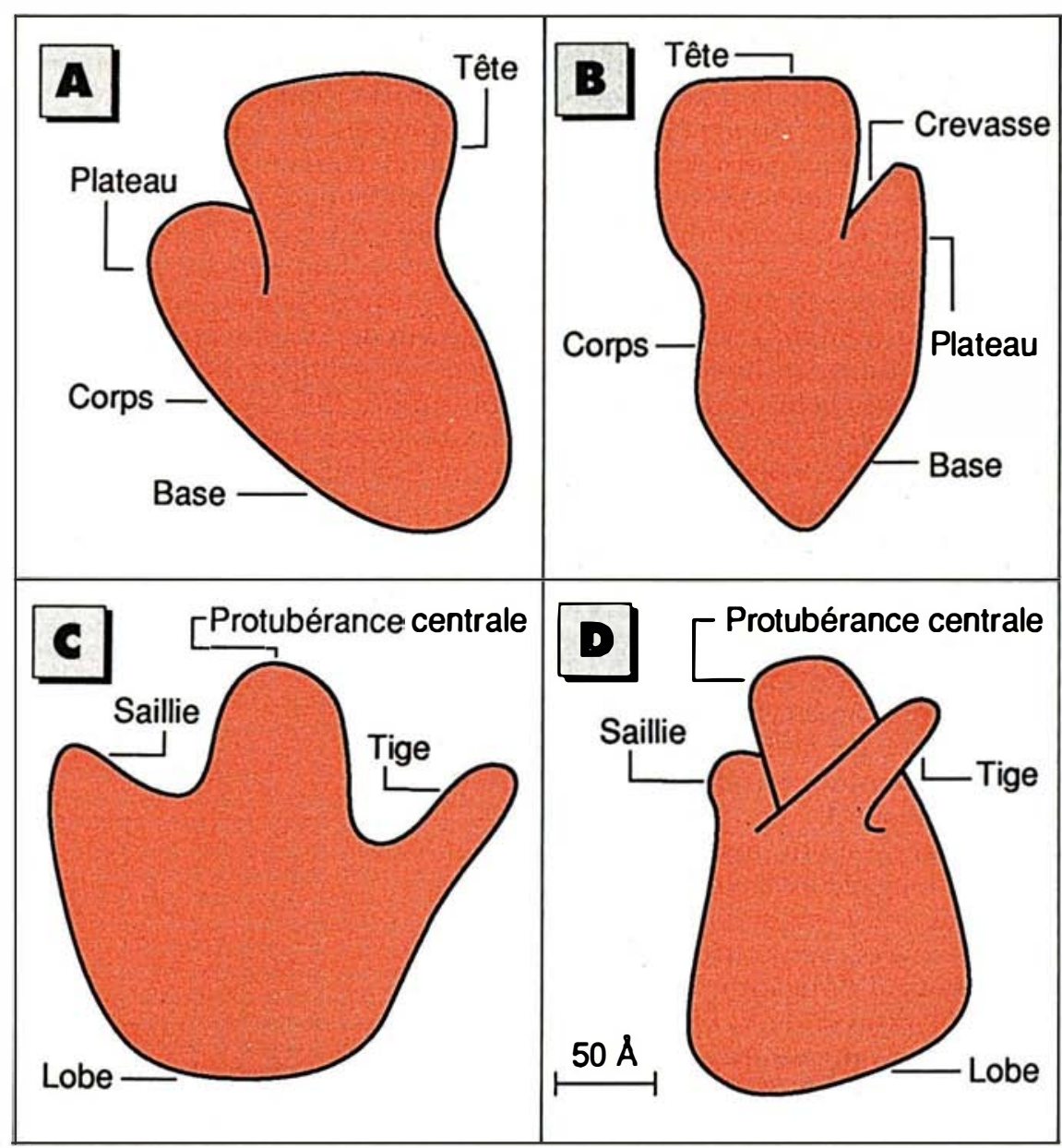

Figure 2. Modèle consensus des sous-unités ribosomales de E. Coli. Les sous-unités ribosomales de $E$. coli sont représentées à l'aide de projections en deux dimensions. La sous-unité 30S, face externe (A) et vue de profil (B). La sousunité 50S, face interne (C) et vue de profil (D). (D'après [11] et [12].)

chiffre (de Sl à S2l et de Ll à L34). La lettre $S$ ou $\mathrm{L}$ indique leur provenance $(\mathrm{S}$, la petite sous-unité et $\mathrm{L}$, la grande sous-unité) (voir Tableau I). Chaque protéine est représentée par une copie unique à l'exception de L7 et L12, qui ne diffèrent que dans leurs extrémités $\mathrm{N}$-terminales (le groupement $\mathrm{NH}_{2}$ de $\mathrm{L} 7$ est acétylé). Il existe en réalité deux dimères L7/L12. Signalons enfin que la protéine L26 est identique à S20. Toutes les protéines ribosomales de $E$. coli ont été séquencées et font l'objet d'une série d'études visant à élucider leur structure tridimensionnelle ainsi que leur rôle fonctionnel [2, 3]. Elles sont généralement riches en résidus basiques $(20 \%$ de lysine + arginine).

L'autre constituant du ribosome, qui intervient pour environ les deux tiers de la masse totale, est de l'acide ribonucléique (ARNr). La sous-unité $30 \mathrm{~S}$ de E. coli renferme une molécule d'ARNr 16S ; la sous-unité $50 \mathrm{~S}$ en contient deux, un ARNr 23S et un 5S (80\% de la masse totale en acide ribonucléique d'une bactérie est contenu dans ses ribosomes). Le Tableau I reprend quelques propriétés physiques des différents constituants du ribosome de E. coli. Les séquences d'une centaine d'ARNr $16 \mathrm{~S}$, d'une cinquantaine de $23 \mathrm{~S}$ et de plus de cinq cents ARNr 5S, provenant des organismes les plus divers, sont de nos jours disponibles [4]. Plusieurs bases de l'ARN 16 et $23 \mathrm{~S}$ contiennent des modifications posttranscriptionnelles qui apparaissent dans des régions conservées, suggé- 
Tableau I

CARACTÉRISTIQUES PHYSIQUES DU RIBOSOME DE E. coli ET DE SES SOUS-UNITÉS

\begin{tabular}{|l|c|c|c|}
\hline & Ribosome & Petite sous-unité & Grande sous-unité \\
\hline Coefficient de sédimentation & $70 S$ & $30 S$ & $50 S$ \\
Masse (kDa) & 2520 & 930 & 1590 \\
\hline Nombre de bases des ARNr & 4565 & $1541(16 \mathrm{~S})$ & $2904(23 S)$ \\
Masse des ARNr (kDa) & 1664 & 560 & $120(5 S)$ \\
Proportion (\%) & 66 & 60 & 70 \\
\hline Nombre de polypeptides & 53 & 21 & 32 \\
Masse totale (kDa) & 857 & 370 & 487 \\
Proportion (\%) & 34 & 40 & 30 \\
Dimensions (nm) & & $5,5 \times 22 \times 22$ & $15 \times 20 \times 20$ \\
\hline
\end{tabular}

rant qu'elles jouent un rôle fonctionnel important. De nombreux travaux ont mis l'accent sur la grande similitude des structures secondaires des ARNr issus d'espèces très éloignées. La comparaison des séquences fortement conservées au cours de l'évolution semble se révéler un outil indispensable en phylogenèse [5]. Divers modèles de la structure secondaire des ARNr 5, 16 et $23 S$ de $E$. coli ont été proposés [6-8]. Un modèle consensus des ARNr 5 et $16 \mathrm{~S}$ de $E$. coli se dégage de ces travaux, alors qu'un désaccord subsiste quant à la structure secondaire de l'ARNr 23S [9]. Une discussion très détaillée des différences et similitudes qui existent entre ces modèles fait l'objet de deux travaux importants $[6,10]$.

\section{L'architecture ribosomale}

Un intense travail de recherche en microscopie électronique a été consacré à la morphologie des sous-unités et des ribosomes entiers. Nous avons repris dans les figures 2 et 3 le modèle consensus du ribosome de $E$. coli et de ses sous-unités [11, 12]. Il en existe toutefois d'autres, qui varient dans le degré d'asymétrie des sous-unités ou dans certains détails structurels, comme la profondeur de certaines dénivellations ou la forme de quel- ques protubérances (voir les deux revues récentes [13, 14]). Notons également l'apparition de techniques de reconstruction d'images en trois dimensions, à partir de micrographies réalisées à l'aide d'un bombardement électronique de faible intensité $[15,16]$. Les micrographies de ribosomes provenant de cellules eucaryotiques montrent une grande similitude de structure avec les ribosomes procaryotiques, en dépit d'un nombre plus grand de consti- tuants [12, 17]. La méthode de choix, permettant une analyse approfondie des détails structuraux serait la cristallographie aux rayons $\mathrm{X}$; malheureusement, les cristaux de ribosomes de $E$. coli obtenus à ce jour sont très difficilement exploitables [18].

\section{L'arrangement spatial des constituants}

La connaissance détaillée des différents constituants du ribosome est

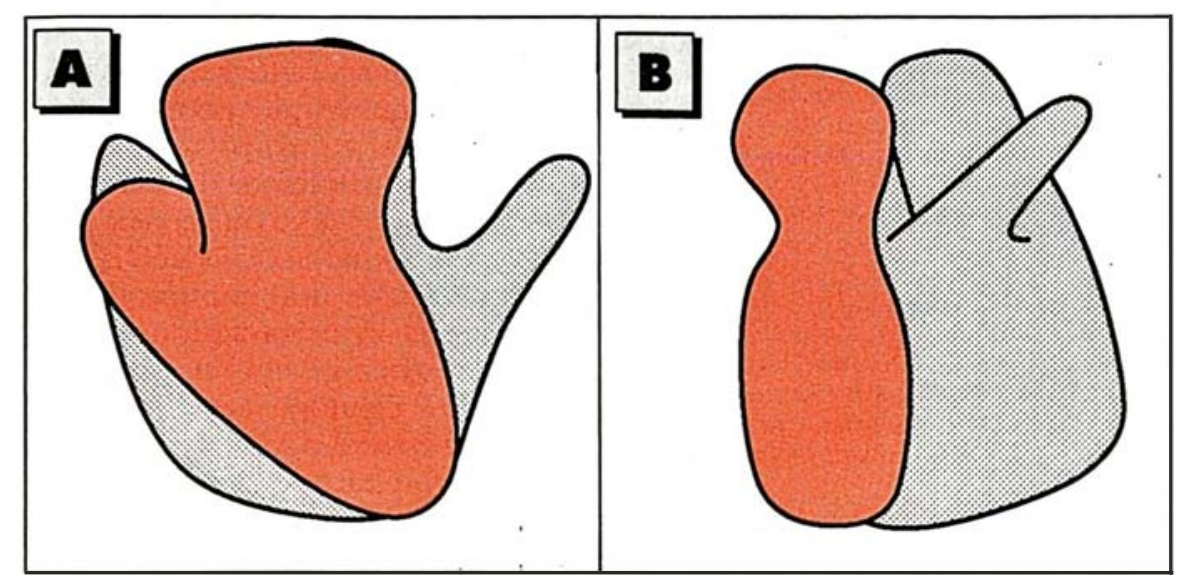

Figure 3. Modèlo consensus du ribosome de E. Coli. Le ribosome 70S, vues de face (A) et de profil (B). 


\section{RÉFÉRENCES}

13. Liljas A. Structural studies of ribosomes. Prog Biophys Mol Biol 1982; 40 : 161-228.

14. Wittmann HG. Architecture of prokariotic ribosomes. Ann Rev Biochem 1983; 52 : 35-65

15. Rademacker $M$, Wagenknecht $T$, Verschoor A, Frank J. Three dimensional structure of the large ribosomal subunit from E. coli. EMBO J 1987 ; 6 : 1107-14.

16. Hoppe W, Oettl H, Tietz HR. Negatively stained 50S ribosomal subunits of $E$. coli. J Mol Biol 1986 ; 192 : 291-322.

17. Boublik M, Hellmann W, Jenkinks F Structural homology of ribosomes by electron microscopy. Proc 10th International Congres on Electron Microscopy. Hamburg : 1982 : 95 6.

18. Yonath AE, Bartunik HD, Bartels KS, Wittmann HG. Some X-ray diffraction patterns from -single crystals of the large ribosomal subunit from B. stearothermophilus. J Mol Biol 1984; 177 : 201-6.

19. Lake JA. Ribosome structure and functional sites. In : Chamblis G, et al., eds. Ribosomes, structure, functions and genetics. Baltimore: University Park Press, 1980 : 207-36.

20. Stöffler G, Stöffler-Meilicke M. Immuno electron microscopy of ribosomes. Ann Rev Biophys Bioeng 1984 ; 13 : 303-30.

21. Capel MS, Kjeldgaard M, Engelman DM, Moore PB. Positions of S2, S13, S16, S17, S19 and $\mathrm{S} 21$ in the $30 \mathrm{~S}$ ribosomal subunit of Escherichia coli. J Mol Biol 1988 ; 200 : 65-87.

22. TrautRR, TewariDS, SommerA. Gavino GR, Olson HM, Glitz DG. Protein topography of ribosomal functional domains. In : Hardesty B, Kramer G, eds. Structure, function and genetics of ribosomes. New York: Springer-Verlag, 1986 : 206-308.

23. Pellegrini M, Cantor CR. Affinity labeling of ribosomes. In : Weissbach $\mathrm{H}$, Pestka S eds. Molecular mecanisms of protein biosynthesis. New York : Academic Press, 1977 : 203- insuffisante pour définir le mode de fonctionnement de celui-ci. Une batterie de techniques a donc été mise en œuvre dans le but de définir la position et la fonction de chaque composant au sein de la structure globale. Les techniques les plus couramment utilisées ont été: (1) la microscopie électronique couplée à des méthodes immunologiques, une technique utile pour la localisation de protéines ribosomales ou de portions d'ARNr facilement accessibles à la surface des sous-unités ou du ribosome entier [19, 20]; (2) la diffusion de neutrons qui permet de mesurer la distance entre les centres de masse des différents constituants[21]; (3) le pontage chimique, réalisé à l'aide de divers agents bifonctionnels, entre protéines voisines, particulièrement celles qui se situent à l'interface des deux sous-unités, au sein de la particule entière $[22,23]$ soit entre les ARNr et les protéines [24, 25] ainsi qu'au sein même des ARNr [26] ; (4) la mesure des distances entre groupements fluorescents placés à des sites spécifiques (mesure du transfert d'énergie entre deux fluorophores). Cette technique a été utilisée avec succès dans le but de mesurer les distances entre les extrémités 3' des ARNr [27] ou entre divers points situés sur deux ARN de transfert positionnés dans leur site respectif, A et $\mathrm{P}[28]$; (5) la construction de cartes d'assemblage (ordre dans lequel les protéines ribosomales s'associent aux ARNr) a permis d'obtenir des renseignements topographiques très précieux [29, 30]. Il existe une bonne corrélation entre le scénario d'assemblage des sous-unités à partir des composants isolés et la proximité physique de ceux-ci au sein du ribosome entier. Les techniques immunologiques permettent de produire des anticorps spécifiques (immunoglobulines de la classe $\mathrm{G}$ ) contre des portions (épitopes) de protéines ribosomales ou d'ARNr. Le site de liaison des immunoglobulines, mis en évidence au microscope électronique, révèle la position qu'occupe l'épitope au sein de la particule entière. Grâce à cette technique, la plupart des protéines de la sous-unité $30 \mathrm{~S}$ ainsi qu'une quinzaine de la grande sous-unité $50 \mathrm{~S}$ de $E$. coli ont été localisées (figure 4). Elle a en outre permis de positionner les extrémités 3' des différents $\mathrm{ARNr}$ l'extrémité 5' de l'ARNr 16S, ainsi que quelques bases «modifiées» contenues dans ce dernier (la méthylguanine en position 526 ou les adénines diméthylées en position 1517 et 1 518) [6]. La diffusion de neutrons utilise la possibilité de reconstituer, à partir des composants isolés, des sous-unités ribosomales parfaitement fonctionnelles. Lors de la reconstitution, une paire de protéines dont on veut mesurer la distance est remplacée par les mêmes protéines deutérées. En variant la proportion de $\mathrm{D}_{2} 0$ dans le solvant, on peut «effacer» Ia contribution des protéines hydrogénées à l'intensité diffusée. Le faisceau de neutrons ne «voyant» alors que les protéines deutérées, on peut estimer la distance entre les centres de masse de ces protéines. En mesurant la distance de différentes paires, et par triangulations successives, on obtient une idée de la localisation relative de chaque protéine au sein de la sous-unité [21]. Il est assez remarquable de constater que les résultats provenant des différentes techniques décrites ci-dessus s'accordent sur la position relative d'une vingtaine de protéines de la sous-unité $30 \mathrm{~S}$. Un modèle très détaillé de cette sous-unité, qui intègre toutes les données accumulées ces dix dernières années, vient d'être présenté [25].

\section{Topographie des centres actifs}

Si quelques fonctions ribosomales semblent parfois impliquer l'une ou l'autre protéine ou une portion d'ARNr, aucune n'a pu, à ce jour, être reproduite par un constituant ou groupe de constituants isolés.

- Les sites de décodage et de liaison de l'ARNm. L'ARNm se lie spécifiquement à la sous-unité $30 \mathrm{~S}$. Les protéines indispensables à cette liaison sont S1, S3, S18, et S21 et ont été localisées dans la partie supérieure droite de l'interface des deux sousunités (figures $4 A$ et $4 B$ ). Dans cette même région de la sous-unité $30 \mathrm{~S}$ on retrouve également les sites de liaison des trois facteurs de démarrage IFl, 2,3 [31]. Le pontage chimique, réalisé entre un nucléotide de l'anticodon d'un ARNt placé au 


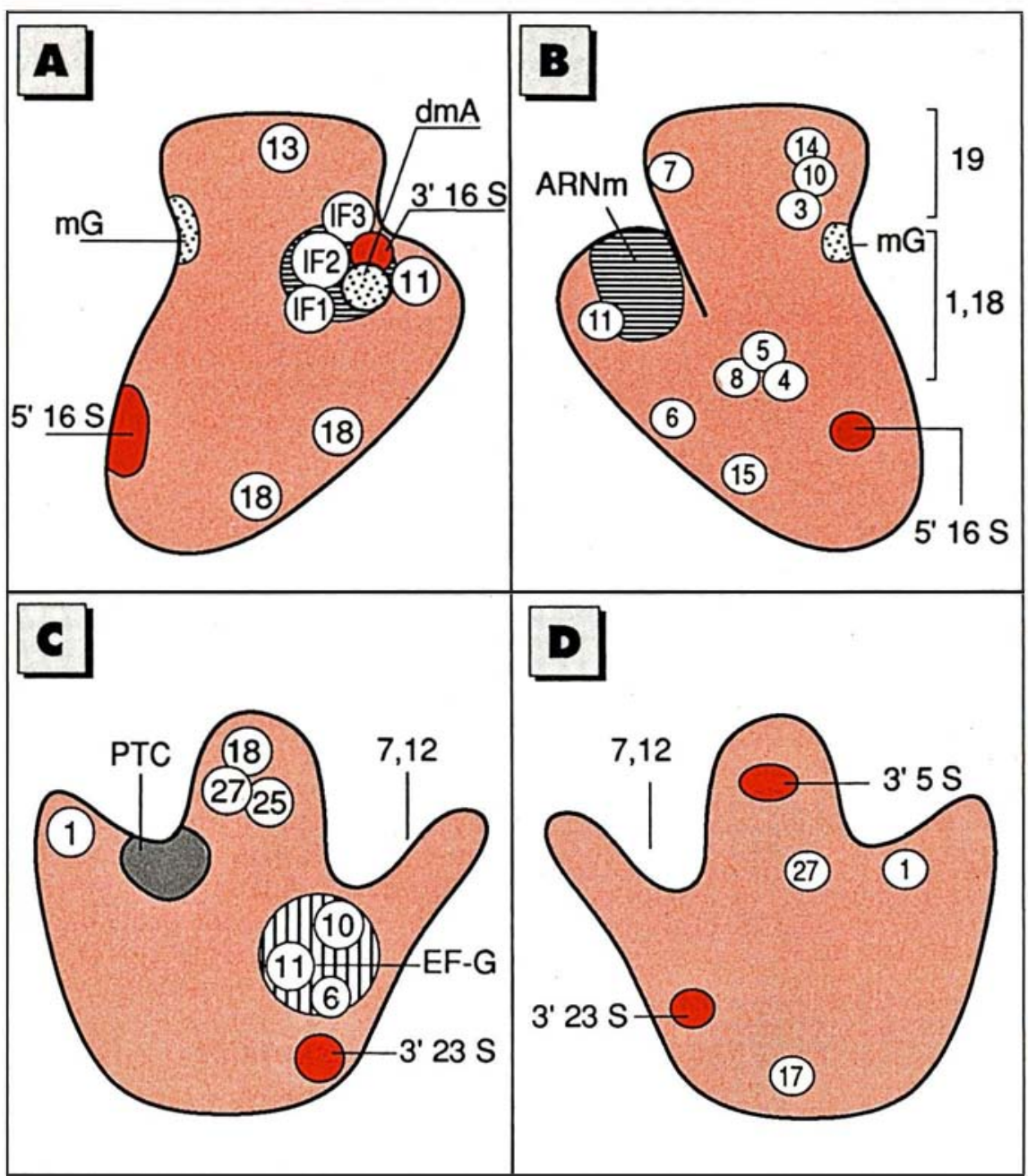

Figure 4. Localisation de certains constituants et sites actifs. L'emplacement des chiffres encerclés donne la position des protéines ribosomales déterminée par microscopie électronique. (A) Vue de la sous-unité 305 faisant face à la grande sous-unité. IF1, IF2 et IF3 indiquent le site de liaison des facteurs de démarrage. Le site de liaison de l'ARNm (site de décodage) est représenté par la surface hachurée horizontalement. mG est la méthyl-guanine de l'ARNr 16S (position 526); dmA les deux diméthyl-adénines (positions 1517 et 1518). Les positions des extrémités $3^{\prime}$ et $5^{\prime}$ de l'ARNr 165 sont également indiquées. (B) Vue de la face externe de la sous-unité $30 S$. (C) Vue de la sous-unité $50 S$ faisant face à la petite sous-unité. PTC = domaine de la peptidyl-transférase. Le site de liaison du facteur d'élongation G (centre d'hydrolyse du GTP) est schématisé par la surface hachurée verticalement. (D) Vue de la face externe de la sous-unité 505.

site $\mathrm{P}$ et la cytosine 1400 de l'ARNr $16 \mathrm{~S}$, démontre la proximité de ce dernier au site de décodage [32]. La cytosine 1400 est proche de l'extrémité 3' de l'ARNr 16S, laquelle interagit directement avec une séquence particulière, dite de Shine-Dalgarno, contenue dans l'ARN messager [33]. De plus, cette base a été localisée, par microscopie électronique, dans la même région que les protéines citées $\mathrm{m} / \mathrm{s} n^{\circ} 9$ vol. 5 , novembre 89 qui plaide en faveur d'un recouvrement partiel de leurs sites de liaison. Le thiostrepton, un antibiotique qui inhibe cette liaison, interagit avec la protéine Lll et un fragment d'ARNr 23S [34]. En outre, des pontages chimiques ont montré que les protéines L7 et L12 étaient communes aux deux sites. Des ribosomes dépourvus de ces protéines sont inaptes à réaliser les différentes réactions d'hydrolyse du GTP au cours du processus de traduction. Notons enfin que les protéines L7 et L12 provenant d'organismes aussi différents que procaryotes et eucaryotes ont des séquences fortement conservées. Celles de E. coli, par exemple, sont capables de restaurer la capacité de synthèse de ribosomes d'eucaryotes dont les protéines équivalentes ont été préalablement enlevées. Ces observations suggèrent l'emplacement des sites de EF$G$ et EF-Tu à l'interface des sousunités, à la base de la tige formée par L7 et L12 (figure 4C).

- Le domaine de la peptidyl-trans férase. Cette réaction enzymatique se déroule exclusivement sur la grande sous-unité : en fait, elle peut se mesurer en l'absence de la petite sousunité. Le marquage d'affinité du ribosome, à l'aide de divers $\mathrm{ARNt}$ modifiés à leur extrémité 3' (extrémité qui reçoit l'acide aminé ou le peptide) ainsi que de dérivés d'antibiotiques interférant avec la formation du lien peptidique, implique six à sept protéines de la sous-unité $50 \mathrm{~S}$ et deux de la sous-unité $30 \mathrm{~S}$ [23]. Lorsque l'ARNt est placé au site A, le marquage révèle la présence de la protéine $\mathrm{Ll} 6$ et dans une moindre mesure celle de L15, L2 et L27. En revanche, le site $P$ serait essentiellement formé par les protéines L2 et L27. Ces protéines (notamment L27 et L15) sont localisées à la base et à gauche de la protubérance centrale de la sous-unité $50 \mathrm{~S}$ (figure $4 C$ ). Ces résultats sont confirmés par la visualisation directe du site de liaison de la puromycine grâce à la microscopie électronique [35]. Rappelons que cet antibiotique se lie au site accepteur de la peptidyl-transférase. Signalons enfin que l'uracile, en position 2585 de l'ARNr 23S [36], semble également faire partie intégrante du domaine de la peptidyl-transférase. 


\section{RÉFÉRENCES}

24. Greuer B, Osswald M, Brimacombe R, Stöffler G. RNA-protein cross-linking in E. coli $30 \mathrm{~S}$ ribosomal subunits; determination of sites on 16S RNA that are cross-linked to proteins S3, S4, S7, S9, S10, S11, S17, S18 and $S 21$ by treatment with bis-(2-chloroethyl)-methylamine. Nucleic Acids Res 1987 ; $15: 3241-55$.

25. Brimacombe R, Atmadja J, Stiege W, Schuler D. A detailed model of the threedimensional structure of E. coli $16 S$ ribosomal RNA in situ in the $30 \mathrm{~S}$ subunit. $\mathrm{J} \mathrm{Mol}$ Biol 1988; 199 : 115-36.

26. Ofengand J, Gornicki P, Nurse K, Boublik M. On the structural organisation of the tRNA-ribosome complex. In: Clark BFC, Pedersen HU, eds. Gene expression : the translational step and its control. Copenhagen: Munksgaard, 1984 : 293-315.

27. Odom OW, Robbins DJ, Dottavia-Martin D, Kramer G, Hardesty B. Distances between '3' ends of ribosomal ribonucleic acids reassembled into $E$. coli ribosomes. Biochemistry 1980 ; 19 : 5947-54.

28. Paulsen H, Robertson JM, Wintermeyer $W$. Topological arrangement of two tRNAs on the ribosome. Fluorescence energy transfer measurements between $A$ and $P$ site bound IRNA. J Mol Biol 1983 ; 167 : 411-26.

29. Traub P, Nomura M. Structure and func tion of $E$. coli ribosomes. Reconstitution of functionnally active $30 \mathrm{~S}$ ribosomal particles from RNA and proteins. Proc Natl Acad Sci USA 1968 ; 59 : 777-84.

30. Dohme F, Nierhaus KH. Total reconstitution and assembly of $50 \mathrm{~S}$ subunits from E. coli ribosomes in vitro. J Mol Biol 1976 ; $107: 585-99$

31. Boileau G, Butler P, Hershey JWB Traut RR. Direct cross-links between initiation factors 1,2 and 3 and ribosomal proteins promoted by 2 -iminothiolane. Biochemistry 1983 ; 22 : 3162-70.

32. Ofengand J, Ciesiolka J, Nurse K. Ribosomal RNA at the decoding site of the tRNAribosome complex. In: Hardesty B, Kramer G, eds. Structure, function and genetics of ribosomes. New York: Springer Verlag, 1986 : 473-94.

33. Shine J, Dalgarno L. The 3' terminal sequence of $E$. coli $16 \mathrm{~S}$ ribosomal RNA : complementarity to nonsense triplets and ribosome binding sites. Proc Natl Acad Sci USA 1974 ; 71 : 1342-6.
34. Thomson J, Cundliffe E, Stark M. Binding of thiostrepton to a complex of 23S rRNA with protein Lll. Eur J Biochem 1979; $98: 261-5$.

35. Olsen HM, Nicholson AW, Cooperman BS, Glitz DG. Localization of sites of photoaffinity labeling of the large subunit of $E$. coli ribosomes by an arylazide derivative of puromycin. J Mol Chem 1985; 260 : 1032631.

36. Ofengand J. The topography of IRNA binding sites on the ribosome. In: Chamblis G, et al., eds. Ribosomes, structure, function and genetics. Baltimore: Park Press, 1980 : 497-529.

37. Ryabova LA, Selivanova OM, Baranov VI, Spirin AS. Does the channel for nascent peptide exist inside the ribosome? FEBS Lett 1988; 226 : 255-60.

38. Vasiliev VD, Selivanova OM, Baranov VI Spirin AS. Structural study of translating $70 S$ ribosomes from E. coli. FEBS Lett 1983; 1557: 167-72.

39. Dahlberg A. Site directed mutagenesis of E. coli rRNA. In : Hardesty B, Kramer G, eds. Structure, function and genetics of ribosomes. New York: Springer Verlag, 1986 : 686-98.

40. Thompson JF, Hearst JE. Structure-function relations in E. coli $16 S$ RNA. Cell 1983 ; 33 : 19-24.

41. Dabbs ER. Selections for E. coli mutants with proteins missing from the ribosome. $J$ Bacteriol 1979 ; 140 : 734-7.

42. Kruger K, Grabowski P J, Zang A J, Sands J, Gottschling DE, Cech TR. Self-splicing RNA. Cell 1982 ; 31 : 147-57.

43. Zaug AJ, Cech TR. The intervening sequence RNA of Tetrahymena is an enzyme. Science 1986 ; 231 : 470-5.

44. Sharp PA. On the origin of RNA splicing and introns. Cell $1985 ; 42$ : $397-400$.

45. Guerrier-Takada C, Gardinier K, Marsh T, Pace N, AltmanS. The RNA moiety of ribonuclease $P$ is the catalytic subunit of the enzyme. Cell 1983 ; 35 : 849-57.

46. Moazed D, Noller HF. Interaction of antibiotics with functional sites in $16 \mathrm{~S}$ ribosomal RNA. Nature 1987 ; 327 : 389-94.

47. Sigmund CD, Ettayebi M, Morgan EA Antibiotic resistance mutations in $16 S$ and 23S ribosomal RNA genes of E. coli. Nucleic Acids Res 1984; 12 : 4653-63.
- Les sites de liaison des ARNt. De nombreux travaux ont été consacrés à l'agencement des deux sites de liaison des ARNt [26, 36]. La proximité des deux anticodons (au site de décodage) et celle des extrémités aminoacyl et peptidyl (dans le domaine de la peptidyl-transférase) sont difficiles à concilier, compte tenu de la structure tridimensionnelle cristalline des ARNt. Un modèle, récemment proposé, place les deux ARNt dans une cavité à l'interface des deux sousunités [32].

- Tunnel de sortie du polypeptide en formation. L'hypothèse, longtemps admise, de l'existence entre les deux sous-unités d'un tunnel, assurant le cheminement de la chaîne peptidique en croissance, a été remise en cause récemment [37].

Le site de sortie de l'extrémité $\mathrm{N}$ terminale d'un peptide en croissance se localiserait plutôt dans une poche située entre la base de la protubérance centrale et la saillie formée par Ll; le peptide serait acheminé le long d'un sillon situé sur la face externe de la sous-unité $50 \mathrm{~S}$.

\section{Conclusions et perspectives}

Depuis une vingtaine d'années, des efforts considérables ont été investis pour mieux comprendre les mécanismes qui régissent la biosynthèse des protéines. La structure primaire (et parfois secondaire) de la plupart des macromolécules qui composent l'appareil de traduction est connue, des modèles intégrant les divers centres actifs dans le cadre général de la structure tridimensionnelle du ribosome ont également été proposés [3, $28,32,36,38]$. L'application des techniques de diffraction des $\mathrm{RX}$, de mutagenèse dirigée [39] et de reconstruction d'images en trois dimensions permettront dans les années à venir d'affiner la structure des composants. Les mécanismes des réactions biochimiques qui accompagnent la translocation (éjection de l'ARNt désacylé, transfert du peptidyl-ARNt au site $\mathbf{P}$ et mouvement simultané du ribosome le long de l'ARN messager) sont encore peu connus. De même, la fidélité de la traduction ne peut s'expliquer seulement par l'interaction codon-antico- 
don [40]. L'ARNr a longtemps été considéré comme une matrice sur laquelle venaient s'assembler les protéines ribosomales, seules responsables des diverses fonctions enzymatiques du ribosome. Ce rôle passif est remis en question par l'observation qu'un bon nombre de protéines n'est pas indispensable pour la traduction [41]. D'autre part, certaines molécules d'ARN semblent être dotées d'activité enzymatique, sans l'assistance d'une quelconque protéine. En effet, au cours de l'étape de maturation de certains ARN, l'excision d'un intron et l'épissage des exons peuvent être catalysés par l'ARN lui-même [42, 43]. L'autoexcision, observée pour la première fois dans le gène ARNr de Tetrahymena thermophila, ne constitue pas un cas isolé [44]. Citons également le clivage de l'extrémité 5 ' des précurseurs de divers ARN de transfert, produit, sous certaines conditions expérimentales, par la composante ARN de la ribonucléase P [45]. Rappelons enfin que l'analyse comparative révèle dans l'ARNr la présence de séquences nucléotidiques fortement conservées au cours de l'évolution [5]. Ces séquences, qui comptent sans doute parmi les plus anciennes qui existent sur notre planète, constitueraient, au sein du ribosome, des centres actifs importants.

Les résultats des approches biochimiques et génétiques de ce problème abondent dans un sens commun. En effet, les nucléotides de l'ARNr protégés de l'action de divers agents chimiques par la présence d'inhibiteurs de la synthèse protéique, et ceux modifiés chez les mutants résistants à ces mêmes inhibiteurs, sont souvent identiques et se localisent dans les régions fortement conservées au cours de l'évolution [46, 47].

Ces récentes découvertes pourraient avoir d'énormes implications sur les fondements de la biologie moléculaire ainsi que sur notre connaissance de l'origine de la vie. Le modèle classique de l'évolution simultanée des acides nucléiques et des protéines pourrait être abandonné au profit d'un modèle selon lequel l'ARN aurait assumé, dans le stade prébiotique, les fonctions de stockage de l'information et les fonctions nécessaires à sa propre réplication

\footnotetext{
$\mathrm{m} / \mathrm{s} n^{\circ} 9$ vol. 5 , novembre 89
}

\section{Summary}

Bacterial ribosomes : structure and functions

$70 \mathrm{~S}$ ribosomes of procaryotes and eucaryotic organelles are formed of a $50 \mathrm{~S}$ (5S and 23S rRNA plus $32 \mathrm{~L}$ proteins) and a $30 \mathrm{~S}$ (16S rRNA plus $21 \mathrm{~S}$ proteins) subunits, which separate at termination of polypeptide chains and join at initiation (ribosomal cycle). Initiation entails mRNA binding to $30 \mathrm{~S}$; elongation corresponds to peptide bond formation on $50 \mathrm{~S}$; termination produces the dissociation of elongation complexes into their components. Conventional and immune electron microscopy studies have revealed the tridimensional conformation of ribosomal subunits and of $70 \mathrm{~S}$ ribosome, and identified the position of ribosomal proteins. Each rRNA has a specific secondary structure within a subunit, holds sequences binding single proteins or protruding on particle surface, and performs given functions in protein synthesis. The techniques of affinity labeling, neutron scattering and fluorescence energy transfer have contributed to define the topology of ribosome components and functions. The main ribosome function, the peptidyltransfer reaction is carried out by a catalytic center located at the base of the central protuberance of the large subunit : it is made of a 23S rRNA loop and of few $\mathrm{L}$ proteins. This center is covered by the small subunit : at the interface between the subunits are, in fact, located the binding sites for mRNA, two aminoacyl-tRNA (A and $P$ sites) and the elongation factors EF-Tu and EF-G.

\section{TIRÉS A PART}

C. Cocito 